T E C H N I C A L A R T I C LE

\title{
Studies in Composing Hydrogen Atom Wavefunctions
}

\author{
LANCE J. PUTNAM, JOANN KUCHERA-MORIN \\ AND LUCA PELITI
}

The authors present their studies in composing elementary wavefunctions of a hydrogen-like atom and identify several relationships between physical phenomena and musical composition that helped guide the process. The hydrogen-like atom accurately captures some of the fundamental quantum mechanical phenomena of nature and supplies the composer with a set of well-defined mathematical constraints that can create a wide variety of complex spatiotemporal patterns. The authors explore the visual appearance of time-dependent combinations of two and three eigenfunctions of an electron with spin in a hydrogen-like atom, highlighting the resulting symmetries and symmetry changes.

In research laid out in this paper, we have attempted to represent the state of a quantum system and its evolution in an immersive integrated environment so as to present scientists with a better intuitive understanding of quantum reality and composers with a powerful medium for aesthetic investigations and creative expression. The hydrogen-like atom is particularly important for scientists, as it accurately describes some of the fundamental quantum mechanical phenomena of nature. For the composer, this atom supplies a set of welldefined mathematical constraints that can be adjusted to create a wide variety of spatiotemporal structures.

Making such a quantum system perceptible is of utility to both the scientist and the artist. First of all, doing so renders the abstract mathematical system into a format that can be experienced in a more intuitive and visceral way. Such a representation will not necessarily contribute to our understanding of the Hilbert space in which the atom lives but can give a feeling of this abstract space in the more familiar space that we all inhabit. Second, perceptualizing the atom can as-

Lance J. Putnam (academic researcher, educator and composer), Department of Architecture, Design and Media Technology, Aalborg University, Sofiendalsvej 11 , DK-9200 Aalborg, Denmark. Email: <lp@create.aau.dk>.

JoAnn Kuchera-Morin (academic researcher, educator and composer), Media Arts and Technology, University of California, Santa Barbara, Santa Barbara, CA 93106-6065, U.S.A. Email: <jkm@create.ucsb.edu>.

Luca Peliti (academic researcher and educator), Dipartimento di Fisica, Università "Federico II," Complesso Monte S. Angelo, 80126 Napoli, Italy. INFN, Sezione di Napoli, Complesso Monte S. Angelo, 80126 Napoli, Italy. Email: <peliti@na.infn. it>.

See <www.mitpressjournals.org/toc/leon/48/2> for supplemental files associated with this issue. sist in understanding emergent patterns, flows, symmetries and dynamics when multiple hydrogen-like wavefunctions are mixed in superposition. It can be difficult to represent these complex phenomena in one's head from mathematical equations alone.

In order to represent the wavefunctions, we use the AlloSystem software framework [1] to facilitate the interactive visualization of this information and the AlloSphere [2], a spherical three-story virtual reality environment, to allow full immersion. Not only can the AlloSphere provide an intuitive understanding of the hydrogen-like atom through perception [3] but it also permits the observation of multiple levels of structure in a way that is more naturally comprehensible than a flat (wall) display (Fig. 1). The hydrogen-like atom provides a test bed for the AlloSystem software and AlloSphere instrument and a necessary prerequisite for representation of more complex and nuanced quantum systems. We decided, as a first step, to represent the wavefunctions visually, as this appears to be more easily understood than, for instance, translating them into sound.

\section{RELATED WORK}

Over the past century, many approaches have been taken to visualizing atomic orbitals, mostly for didactic purposes. The first visualizations of hydrogen atom orbitals were made in 1931 using time exposure photography of the motions of a modified mechanical lathe [4]. Thaller has done extensive work on the visualization of quantum mechanical states, in particular in three-dimensional systems $[5,6]$ and hydrogenlike atoms, by means of several visualization techniques. However, research emphasizing the temporal evolution of the wavefunctions is comparatively limited.

Several existing interactive tools visualize dynamic mixtures of hydrogen-like atom wavefunctions in real time. Falstad's Hydrogen Atom Applet [7] displays a volume rendering of mixtures of any number of orbitals, but only with low energy levels. Dauger's Atom in a Box [8] also employs volume rendering and allows the mixing of up to eight wavefunctions with relatively high energy levels. These tools, however, do not represent wavefunctions with spin. 


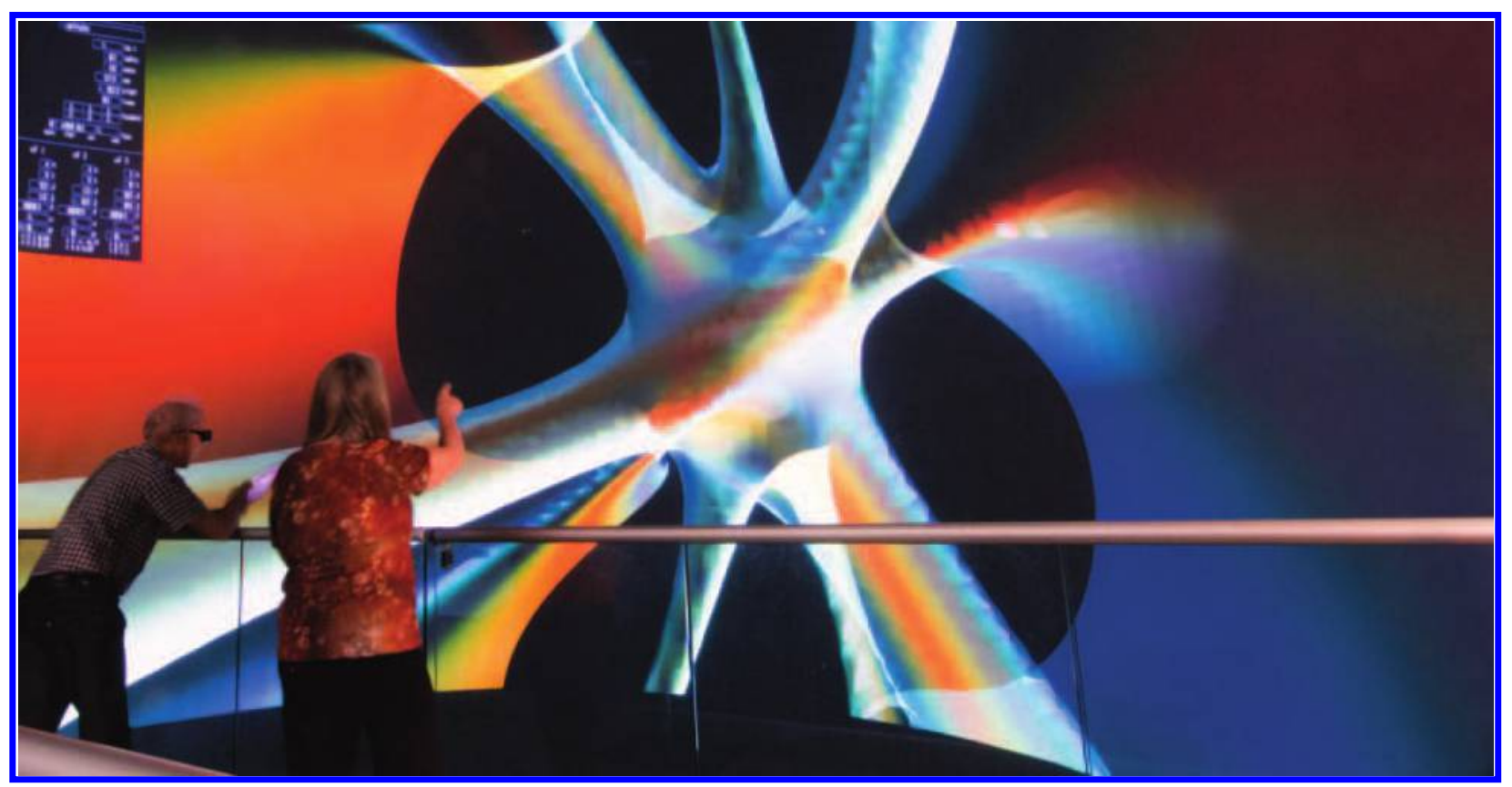

Fig. 1. Researchers controlling parameters of our real-time visualization of a hydrogen-like atom

on the AlloSphere bridge. The AlloSphere is located in the California NanoSystems Institute at the University of California, Santa Barbara. (C) Lance Putnam)

The time evolution of an arbitrary initial wavefunction can be visualized using a software package developed by Belloni and Christian $[9,10]$. The method applies to a onedimensional system with a purely discrete spectrum. The wavefunctions are projected on the eigenfunctions of the system, and subsequent evolution is obtained by evolving the coefficients of the decomposition. While the subtleties of quantum-mechanical evolutions are well captured by this software, its application to higher-dimensional systems would be quite difficult.

Artistically, we identify our work closely with Whitney's concept of digital harmony [11] in applying harmonics and other musical notions, such as scales and transitions of curves, to visual composition. Voss-Andreae's quantum sculptures [12] are also relevant, being visual interpretations of quantum mechanics. However they are more conceptual in nature and do not give a picture of the complex dynamics of quantum systems.

\section{CONCEPTUAL FRAMEWORK}

Our conceptual framework is based on a particular process of musical composition, namely the organization of frequencies and pitches, transposed from the sound domain to the visual domain. More specifically, we propose working with wavefunctions in a way similar to how a composer works with musical tones in creating a larger work.

Quantum mechanics dictates that the evolution of a quantum state is realized by a process that closely resembles additive synthesis in music. A general time-dependent quantum state is obtained by the superposition of a number of wavefunctions representing stationary states-the eigenfunctions-multiplied by periodically varying coefficients. The eigenfunctions correspond to the simple sounds that accrue to form the complete synthesized sound. Their superposition produces the complex spatiotemporal pattern described by the time-dependent wavefunction. Here, we present several concepts in musical composition and physics that support our analogy between wavefunctions and musical tones.

\section{Compositional Process}

Music carries meaning on several timescales, from individual timbres and pitches (foreground) to short melodies and rhythms (middle ground) all the way up to the largescale form and structure of a work (background), each engaging distinct perceptual and cognitive processes. Typically, a composer who starts to realize a piece of music either has a big idea at the macro level (e.g. a large structure to unfold) or something at the micro level, such as a small group of notes. One strategy composers use to master their data in developing a piece is to work at a middle-ground level, that is, controlling the larger structure at the background while unfolding the microstructure at the foreground [13]. Also, in predicting local motion in self-generative music from the microstructure, a middle-ground-to-foreground approach may aid in local directional choices. In Xenakis's compositional approach, local events are decided randomly; however, these events are constrained by clearly articulated probability distributions [14]. This approach is similar to the relationship between the deterministic Schrödinger evolution and probabilistic measurement process in quantum mechanics.

Sketching is a technique used in composition whereby a composer will try out various scenarios of harmonic structures in creating a work. Typically a composer will write a 
series of studies, called études, that are part of the sketching process. The sketching process is hierarchical in that a rough draft of the structure is sketched and various parts are then filled in at the micro level, which will cause adjustments at the macro level. This is the middle-ground area that functions as the pendulum between the micro and macro layers. Since this process is hierarchical, it can be applied at the level of addition of frequencies to build larger structures.

Our initial approach in composing with the hydrogen-like atom was to start with a middle ground, which we identify as interference patterns resulting from mixtures of eigenfunctions, allowing us then to experiment with various combinations of multiple wavefunctions that were scientifically correct and aesthetically pleasing to us. We began the process by sketching a series of studies mixing multiple eigenfunctions together. The studies ranged from the physicsmotivated to the open-ended, exploring beating and complex interference patterns such as fine-splitting. We created the basic musical alphabet by mixing the multiple eigenfunctions together with certain chosen characteristic frequencies.

\section{Eigenfunctions}

We employed eigenfunctions as our basic units of composition. Eigenfunctions are identified by a set of quantum numbers that determine the particular shape of the eigenfunction in space (see Geometric Properties of the Eigenfunctions below) as well as its frequency of evolution through time. Controlling the effect of the quantum numbers on the visual pattern produced is the basic step in the creation of a satisfactory composition.

A quantum state represented by a single eigenfunction evolves through a space-independent phase factor that changes periodically in time with its characteristic frequency. Thus, the spatial distribution of an eigenfunction's magnitude does not change with time. Additionally, two different eigenfunctions can share the same characteristic frequency. An eigenfunction is analogous to a normal mode in a pipe, while a characteristic frequency is analogous to a pure tone.

Eigenfunctions can be placed in superposition to create a wavefunction (see Appendix). Such wavefunctions generally have more complex properties than their constituent eigenfunctions. This is analogous to how more complex tones can be built from simple tones, as occurs when an organ is played, or during musical voicing and chorusing. These emergent complexities often arise due to particular patterns of interference.

\section{Interference Patterns}

From an artistic standpoint, the phenomenon of interference is interesting, as it allows complex emergent patterns to be constructed from an "alphabet" of simple parts. Strong interference patterns occur when two or more waveforms with similar frequencies are superposed.

An elementary form of interference, beating, occurs when two sinusoids with similar frequencies are summed together. Musically speaking, beating effects can enrich static tones by imposing an evolution of timbre relative to pitch that is an order of magnitude slower. To obtain a complex beating pattern, one can mix two waveforms with the same timbre but slightly different pitch. This technique, called celeste, was devised by early organ builders to enrich the sound of individual notes. Similarly, mixing two eigenfunctions produces a spatial pattern that evolves periodically in time, with a frequency equal to the difference between the characteristic frequencies of the eigenfunctions.

While celeste produces a more complex time evolution of a waveform, the effect is strictly periodic in both the time and frequency domains. To alleviate these periodicities, one must mix three or more waveforms together. A wavefunction obtained by superposing three or more eigenfunctions produces a pattern that in general never repeats itself. Interestingly, fine-splitting in an atom can be represented with a mixture of three eigenfunctions.

\section{Hydrogen-Like Atom}

A hydrogen-like atom consists of a single particle of mass $m$ subject to a central force whose potential is inversely proportional to the distance from the nucleus. The characteristic frequencies given by this spectrum rapidly become smaller, leading to slower evolution, as the principal quantum number $n$ increases. They depend only on $n$ and $J$; the $J$ dependence (a spin-orbit coupling effect) is very weak. The $J$ dependence produces the fine-splitting of spectral lines, further discussed in Three Eigenfunctions below.

The hydrogen eigenfunctions provide sufficient complexity to be used as building blocks for our composition. Using hydrogen rather than, say, the harmonic oscillator eigenfunctions is analogous to using an organ to create music rather than individual sine waves. This lends sufficient interest in using the compositional process of mixing waveforms to build chords that act as building blocks for visualization. However, the fact that a great number of eigenfunctions exhibit the same characteristic frequency strongly reduces the number of possibilities to obtain beating patterns. Therefore we took the liberty of assigning frequencies to the eigenfunctions arbitrarily.

\section{Geometric Properties of the Eigenfunctions}

To assist in composition, it is useful to understand the geometrical properties of the eigenfunctions. It is convenient to start from the eigenfunctions of the spinless electron identified by the quantum numbers $(n, \ell, m)$. They are characterized by a number of windings, shells and stacks, i.e. elementary patterns relative to a sphere with a north and south pole on the $z$-axis (Table 1 ). Windings are the cycles made by the phase of the eigenfunction in making one loop around the $z$-axis; stacks are the parallel slices running perpendicular to the $z$-axis; and shells are the concentric regions separated by spherical surfaces where the eigenfunction vanishes.

The eigenfunctions of the electron with spin are obtained by superimposing two spinless eigenfunctions with the same values of $n$ and $\ell$, but with values of $m$ that differ by 1 . Thus, one eigenfunction has one more winding and one less stack than the other. As the spin eigenfunction is identified by a 
TABLE 1. Geometric properties of spinless eigenfunctions in terms of quantum numbers.

\begin{tabular}{|c|c|c|c|}
\hline Parameter & & & Range \\
\hline Winding number & $n_{w}$ & $:=m$ & $\ldots,-2,-1,0,1,2,$. \\
\hline Number of stacks & $n_{s}$ & $:=\ell-|m|+1$ & $1,2, \ldots$ \\
\hline Number of shells & $n_{r}$ & $:=n-\ell$ & $1,2, \ldots$ \\
\hline
\end{tabular}

spinor with two complex components at each point in space, rather than by a single complex number, as in the spinless version, it cannot be simply described in terms of windings and stacks. However, in general, higher quantum numbers correspond to more spatially extended and varying eigenfunctions.

\section{STUDIES}

In our studies, we aimed to explore the emergent structures and dynamics resulting from mixing together multiple eigenfunctions. Due to the vast number of possible eigenfunction combinations, we decided to limit our scope, for now, to mixtures of only two or three eigenfunctions.

To visualize a wavefunction with spin, we used a colormapped isosurface, as we found it gave a clear picture of the wavefunction's overall magnitude and phases. The surface was drawn at a particular value of the wavefunction magnitude $|\Psi|$. The surface was colored according to the spin vector phases $\theta$ (spin-up/-down state) and $\varphi$ (relative phase). We mapped $\theta$ to hues going between orange (spin up) and cyan (spin down) and $\varphi$ to a grayscale gradient. The grayscale gradient is discontinuous, revealing an abrupt cut where the phase completes the cycle [15]. The cuts are more indicative than a smooth mapping and helpful in distinguishing between the two different phases. The hues and grays are interpolated according to the proximity of the spin vector to the equator-more hue near the poles and more gray near the equator (Color Plate D).

\section{One Eigenfunction}

There are already a number of programs allowing for the visualization of single eigenfunctions of the hydrogen-like atom $[16,17]$. Since the time dependence of a single eigenfunction only appears in an overall phase factor, it led to a completely time-independent image in our representation, where the overall phase factor is neglected. Thus the representation of single eigenfunctions lay outside the scope of the compositional process, and we did not investigate it systematically. Indeed, a number of software projects have already tackled the problem of visualizing the eigenfunction of the hydrogen-like atom, both in its spinless version and in that with spin $[18,19]$. We are more interested in the time-dependent patterns one obtains by mixing two or more eigenfunctions.

\section{Two Eigenfunctions}

General Patterns. When mixing two eigenfunctions, we already observe a wide variety of patterns. The patterns evolve periodically, either by changing shape or by rotating, with a frequency proportional to the energy difference of the eigenstates. In order to make this evolution visible, we arbitrarily lower this frequency. Fortunately, these patterns can be classified into a handful of general categories of shapes and dynamics that can be related to the quantum numbers.

We observed the following mutually exclusive types of dynamics with respect to the wavefunction magnitude and spin:

1. No change over time.

2. Rotation around the $z$-axis (with possible exception of relative phase).

3. Beating of magnitude a. isotropically

b. radially

c. along the $z$-axis

Type 1 dynamics involve no change in the wavefunction magnitude and spin vector. This occurs whenever the characteristic frequencies $\in_{v}$ are equal. Type 2 dynamics display a periodic rotation of the wavefunction around the $z$-axis. There is also a periodic change in the relative phase of the spin at the same rate. One can interpret this rotation as an angular traveling wave. Type 3 dynamics are the most interesting and consist of beating effects that may involve a change in the shape of the wavefunction magnitude. The primary constraint on Type 3 dynamics is that the $j$ values are equal. Beating patterns are rotationally invariant around the $z$-axis. For Type 2 and Type 3 dynamics, the rate of change of the wavefunction magnitude is equal to the difference between the two characteristic frequencies. The exact constraints on the eigenfunction parameters are given in Table 2.

The shape of the wavefunction magnitude is limited to a few particular types of point group symmetries. The difference between the $j$ values is the order of dihedral symmetry

TABLE 2. Parameter constraints for two-eigenfunction dynamic types. Empty cells indicate no dependence on the parameter.

\begin{tabular}{|c|c|c|c|c|c|c|}
\hline Dynamic Type & $n$ & $\ell$ & $\boldsymbol{J}$ & i & $\in v$ & Comments \\
\hline 1 & & & & & $=$ & \\
\hline 2 & & & & $\neq$ & $\neq$ & \\
\hline $3(a)$ & $=$ & $=$ & $=$ & $=$ & $\neq$ & \\
\hline $3(b)$ & $\neq$ & $=$ & $=$ & $=$ & $\neq$ & \\
\hline $3(c)$ & & $\begin{array}{l}\ell_{1}+\ell_{2} \\
\text { odd }\end{array}$ & & $=$ & $\neq$ & $\begin{array}{l}\text { Asymmetric } \\
\text { w.r.t } x y \text {-plane }\end{array}$ \\
\hline $3(c)$ & & $\begin{array}{c}\ell_{1}+\ell_{2} \\
\text { even }\end{array}$ & $\neq$ & $=$ & $\neq$ & $\begin{array}{l}\text { Symmetric } \\
\text { w.r.t } x y \text {-plane }\end{array}$ \\
\hline
\end{tabular}



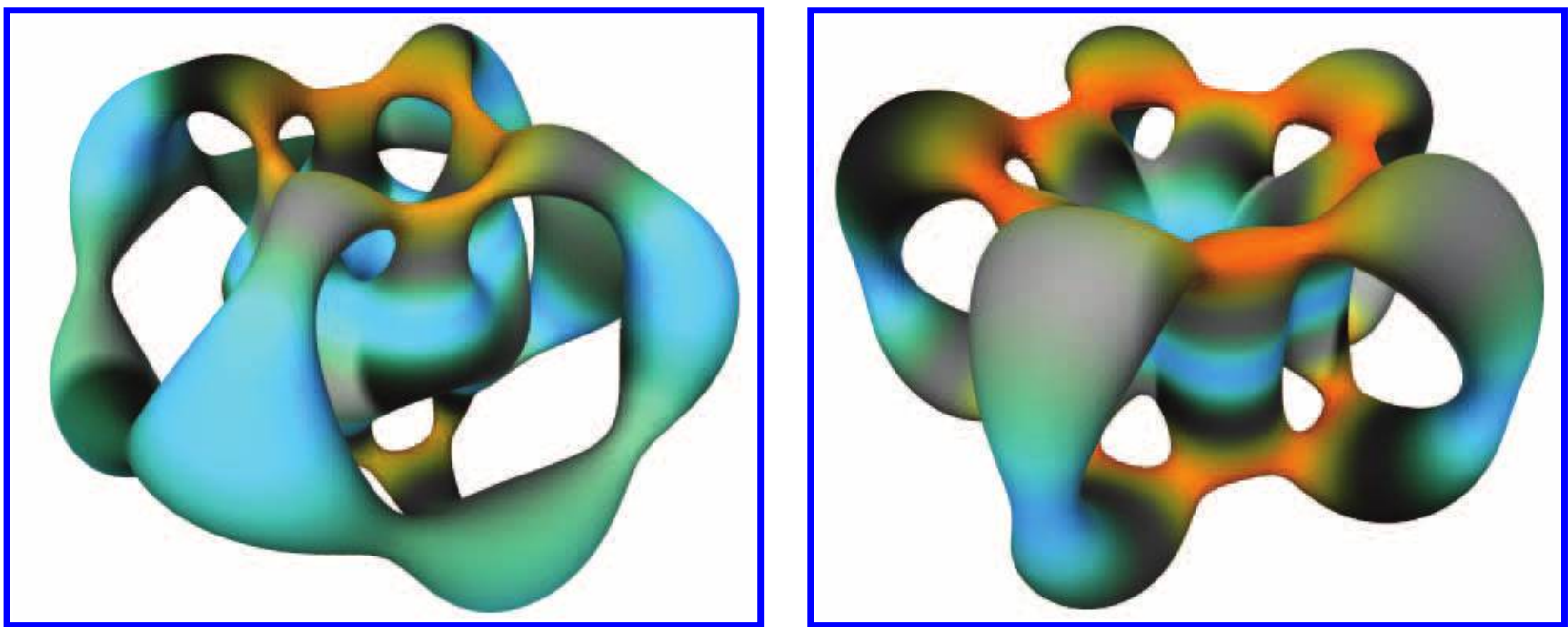

Fig. 2. Two-eigenfunction combinations exhibiting the two possible symmetry types. Here, $\left(n, \ell, J\right.$, i) are $\left(4,2,2 \frac{1}{2},-2 \frac{1}{2}\right)$

and $\left(4,3,3 \frac{1}{2}, i_{2}\right)$. Left: rotation-reflection symmetry with $i_{2}=1 \frac{1}{2}$. Right: reflection symmetry with $j_{2}=2 \frac{1}{2}$. (C) Lance Putnam)

around the $z$-axis, i.e. the number of planes of reflection passing through the $z$-axis. When the $j$ values are equal, there are an infinite number of reflection planes, and thus the shape is rotationally invariant around the $z$-axis. For nonbeating wavefunctions, the shape exhibits either reflection or rotation-reflection symmetry with respect to the $x y$-plane. The rules are

$$
\ell_{1}+\ell_{2}+j_{1}+j_{2} \rightarrow \begin{cases}\text { even } & \text { rotation-reflection } \\ \text { odd } & \text { reflection }\end{cases}
$$

Changing $J$ does not affect symmetry type since it only changes the eigenfunction weights. Complex wavefunctions displaying the two types of symmetry are shown in Fig. 2.

Light-Emitting Combinations. Although we cannot represent the process of light emission in our system, we can illustrate it through a superposition of eigenfunctions that satisfy the selection rules (see Appendix). This leads to a number of interesting wavefunctions that change periodically at a rate equal to the frequency of the emitted photon. Figure 3 shows all such superpositions, which can be built out of the lowest values of $n$. Interestingly, they include all the non-trivial types of dynamics and three distinct types of spin distributions.

When the $n$ values vary widely, one sees a relatively smaller radial interference. Indeed, most of the interference takes place in the region occupied by the eigenfunction with the smaller $n$ value. More complex patterns can be found when the $n$ values are close and larger (Fig. 4).

It appears that the only shapes for light emission superpositions are rotationally invariant around $z$ or have reflective symmetry with respect to the $x y$ plane. None exhibit rotation-reflection symmetry.

\section{Three Eigenfunctions}

Combining three eigenfunctions, each with its own frequency, generally yields a beating pattern that never reproduces itself exactly. The number of possible combinations increases rapidly. We have not yet performed a systematic study of the resulting patterns. As with the simple combinations, the more interesting wavefunctions resulted when we chose values of $n$ close to one another, because, in such cases, the spatial extension of the combining eigenfunctions have a larger overlap.

A special case of physical interest-because it yields the quantum description of a common spectral phenomenon-is an illustration of fine-splitting an emission spectral line. This corresponds to preparing the system in a superposition of three eigenfunctions: a low-energy eigenfunction and two "excited" eigenfunctions (details in Appendix). The resulting wavefunction exhibits a strong beating with a carrier frequency roughly equal to the difference between the frequencies of the excited and the low-lying eigenfunctions, slowly modulated with a frequency equal to the difference between the frequencies of the two excited eigenfunctions. One such combination is shown in Fig. 5. In a real physical situation, the modulation is at least about 18,800 times slower than the beating frequency. Thus, if we were to rescale the involved frequencies faithfully, the modulation would be too slow to be visible. Therefore we chose to rescale the frequencies independently, keeping the modulation frequency somewhat smaller than the fundamental one.

One can explore different combinations of three eigenfunctions in a similar way. Figure 6 shows a combination of higher-quantum number eigenfunctions, which exhibits a slow modulation of the wavefunction shape superimposed on a global rotation. These combinations would apply to, for example, an atom immersed in a magnetic field directed along the $z$-axis.

\section{FUTURE WORK}

\section{Probability Flow}

The evolving shape of the probability distribution is connected with the probability density flow, which demands to be visualized itself, in order to illustrate the nature of the underlying wave dynamics. One could achieve this by a static representation of the velocity field $\mathbf{v}(\mathbf{r}, t)$ or, more intuitively, 


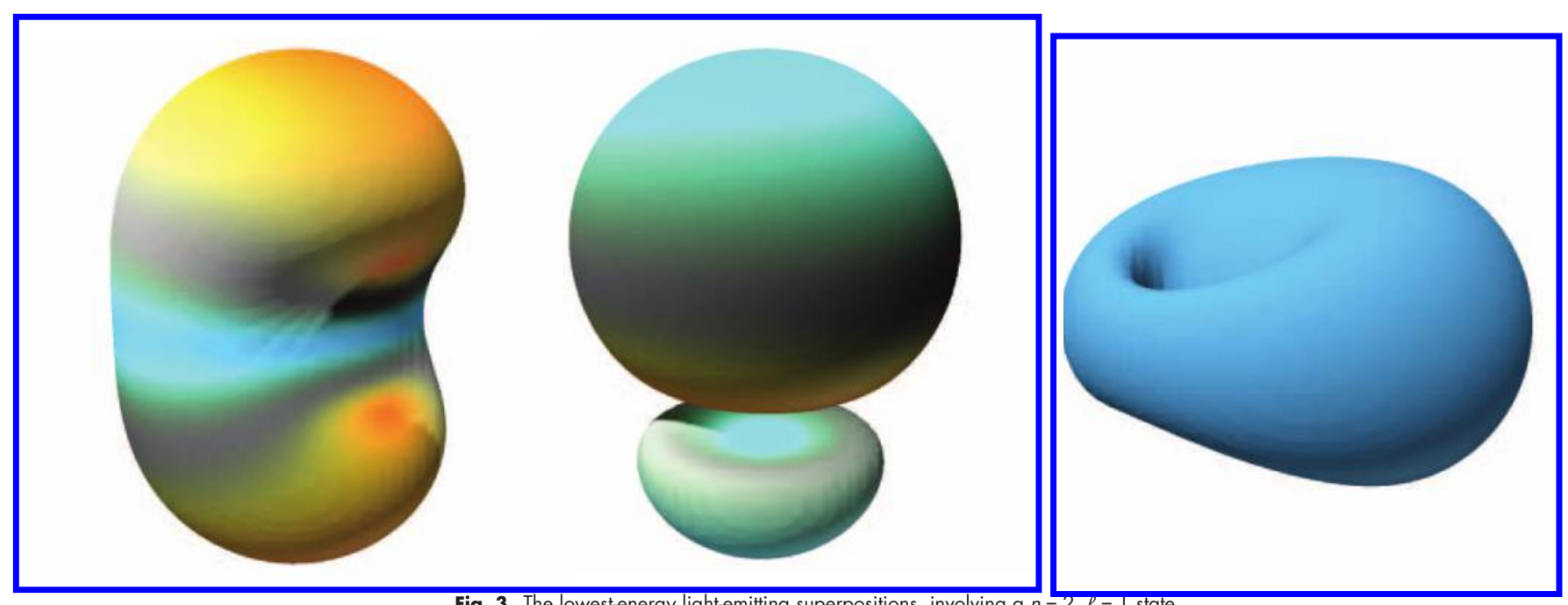

Fig. 3. The lowest-energy light-emitting superpositions, involving a $n=2, \ell=1$ state

and the ground state with $n=1, \ell=0$. (C) Lance Putnam)
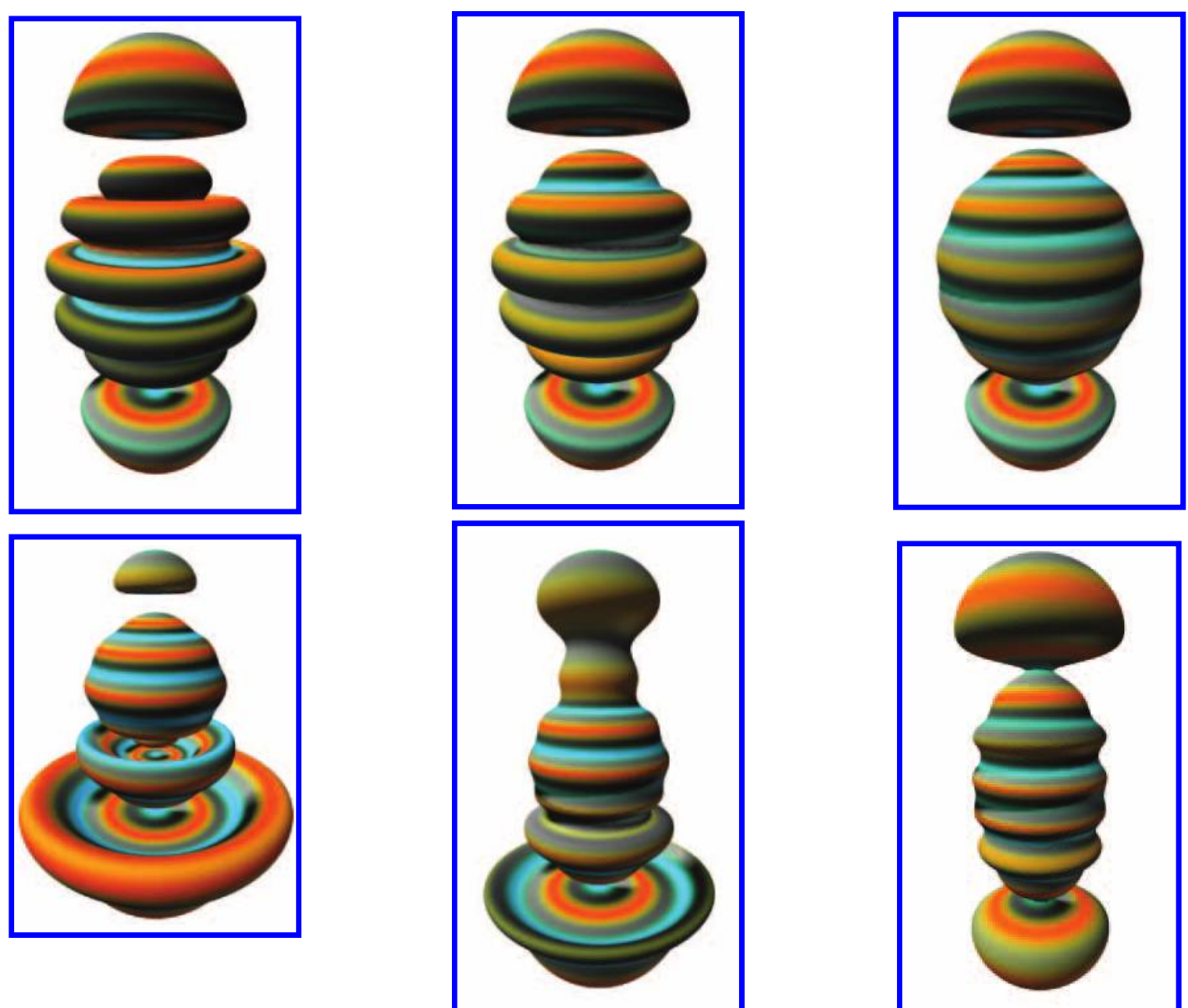

Fig. 4. Beating patterns of IIghi-emiting superpositions with large $n$ values.

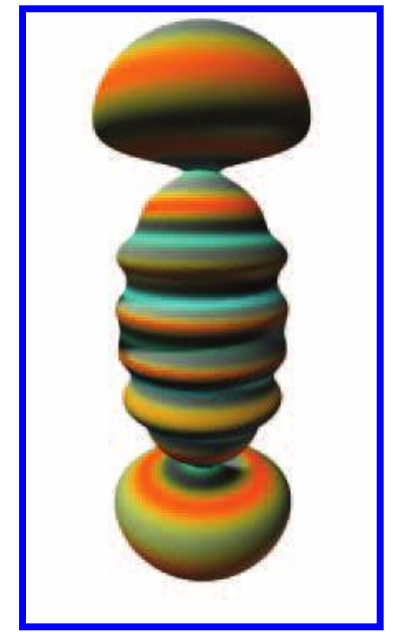

Top row: $n$ values differ by 3 . Bottom row: $n$ values are equal. (C) Lance Putnam) 

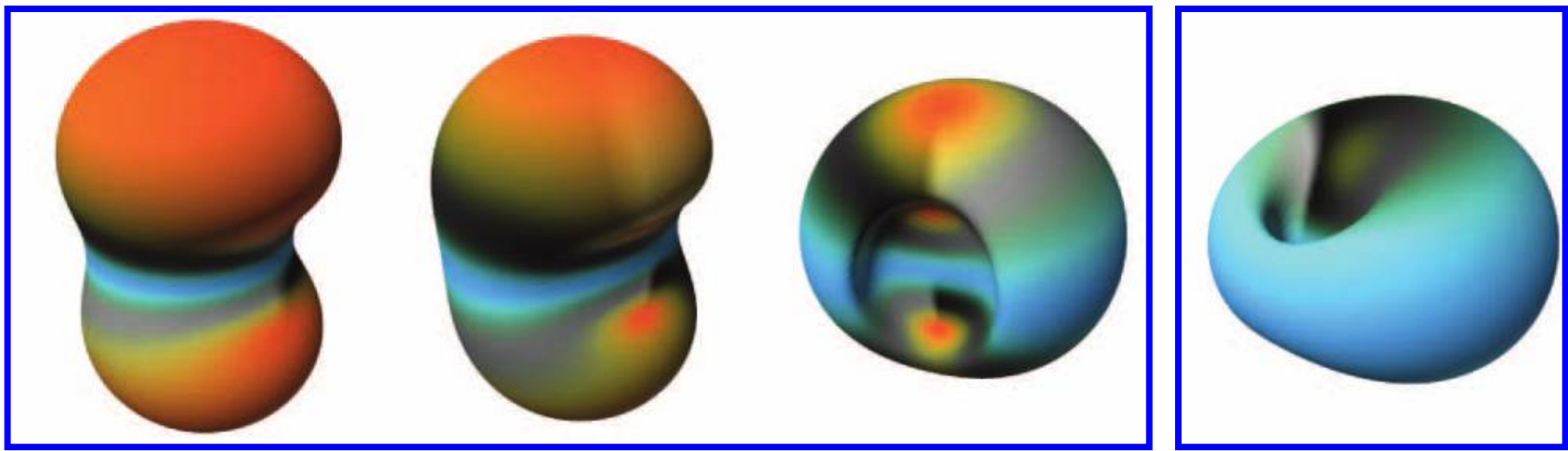

Fig. 5. Time evolution (left to right) of the lowest-energy fine-splitting. One has a combination of an $n=1$

$\ell=0$ ground-state eigenfunction with two excited eigenfunctions having different values of $J$. (@ Lance Putnam)

by representing via flow particles the actual motion with the local velocity. We have explored this option by introducing a small number of agents moving with the local velocity. The dynamics of these agents is connected to the changing shape of the wavefunction in a nontrivial and intriguing way, but a more systematic study is needed.

\section{Time-Dependent Perturbation as a Compositional Process}

In order to introduce a more complex temporal behaviorsuch as transition from a fixed chord to a melody that evolves over time-one could add a time-dependent perturbation $V_{\mathrm{I}}(t)$ to the Hamiltonian. This perturbation changes the wavefunction from, say, a single eigenfunction to a complex superposition of many eigenfunctions. Thus, in principle, one could use a well chosen perturbation to produce a number of different wavefunctions in succession.

There are a number of technical difficulties in following this path. On the one hand, one would need enough computer power to solve the full-fledged time-dependent

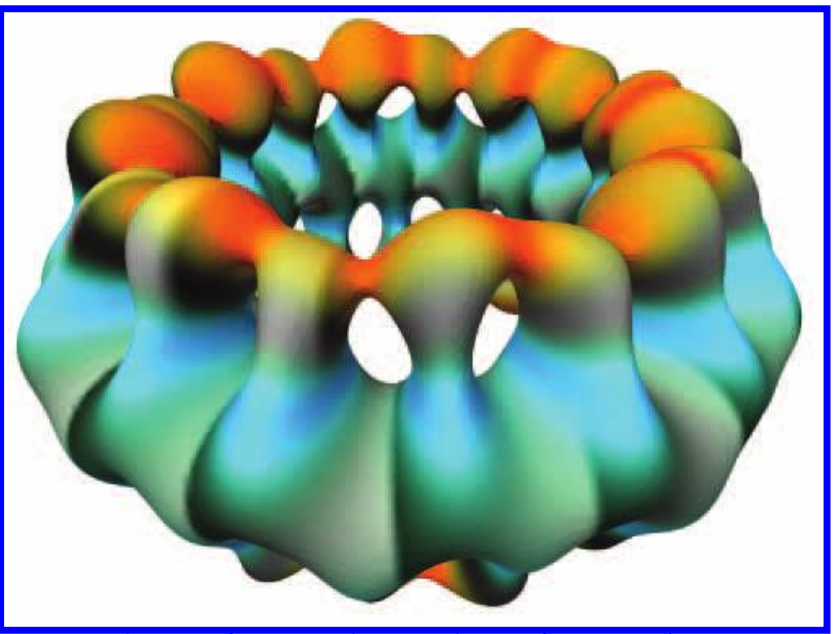

Fig. 6. A three-eigenfunction combination. The eigenfunctions each have $n=10, \ell=9$, and $J=91 \frac{1}{2}$, but three different values of $i\left(71 \frac{1}{2},-91 \frac{1}{2}\right.$ and $-1 / 2$, respectively). The third eigenfunction enters with a small coefficient. This, superposed on the slow rotation of the ensemble, produces a slower modulation of the column heights. (C) Lance Putnam)
Schrödinger equation. One could however make the task more manageable by projecting the evolution back onto a chosen finite-dimensional subspace of wavefunctions, allowing exploitation of the already-implemented representation of the wavefunctions. On the other hand, it is hard to identify which perturbation leads to a given sequence of wavefunctions. While the time-dependence of weights and phases could be chosen arbitrarily, it could be worthwhile to identify physically relevant perturbations that lead to interesting pattern sequences.

\section{CONCLUSION}

With our representation of the hydrogen-like atom, we were able to study various wavefunction combinations that form "chords" from our quantum alphabet of musical "tones." We worked together on a daily basis throughout June 2012 and January 2013, eventually arriving at the current mapping representations. We implemented different iterations of the mappings, most of which we discarded as either not informative enough from the physical point of view or not aesthetically pleasing. For example, we employed point-cloud and vector-field representations of the wavefunction; however, the visual pattern was dominated by the distribution of these reporter glyphs [20]. The isosurface representation has the advantage of leading to an immediate grasp of the broad features of the probability density distribution described by the wavefunction. The subtler effects contained in the spinor amplitudes and phases are reasonably well reported via our color coding. We found with some satisfaction that physics-based combinations often led to aesthetically pleasing patterns, as in the case of the light-emitting superposition.

The interactive tool we built with AlloSystem was easy to use for a visual investigation of the properties of the hydrogen atom eigenfunctions, as a function of their quantum numbers. In this sense alone, the software can be efficiently exploited as a didactic tool. However, even more insight is gained by looking at the unfolding of the visual patterns of a combination of several eigenfunctions. This behavior also provides a more intuitive understanding of the mechanisms of time-dependent perturbation theory. 


\section{APPENDIX}

A hydrogen-like atom wavefunction $\Psi_{v}(\mathbf{r})$ is given as a product of independent radial and angular functions

$\Psi_{\nu}= \begin{cases}R_{n}(r) Y_{\ell m}(\theta, \varphi), & \text { if spin is neglected, } \\ R_{n}(r) Y_{\ell J j}(\theta, \varphi ; s), & \text { with the spin }\end{cases}$

where

For the spinless electron: $v=(n, \ell, m)$;

For the electron with spin: $v=(n, \ell, J, j)$.

Here $(r, \theta, \varphi)$ are the spherical coordinates of the position $\mathbf{r}, s=\uparrow, \downarrow$ is the spin quantum number, and

$n$ is the principal quantum number: $n=1,2,3, \ldots$;

$\ell$ is the angular quantum number: $\ell=0,1, \ldots, n-1$;

$m$ is the magnetic quantum number: $m=-\ell,-\ell+1$,

$\ldots, \ell-1, \ell$;

$J$ is the total angular momentum: $J=\ell \pm 1 / 2$

$j$ is the $z$-component of the total angular momentum:

$$
j=-J,-J+1, \ldots, J-1, J \text {. }
$$

The wavefunctions satisfy the time-dependent Schrödinger equation

$$
\mathrm{i} \hbar \partial_{t} \Psi=\hat{H} \Psi
$$

where the Hamiltonian operator $\hat{H}$ corresponds to the energy of the system. The eigenfunctions $\Psi_{v}$ are solutions of the time-independent Schrödinger equation

$$
\hat{H} \Psi_{v}=\in_{v} \Psi_{v}
$$

and are identified by a collection $v$ of quantum numbers. A general time-dependent wavefunction $\Psi$ is obtained as a linear combination of eigenfunctions with time-varying coefficients $c_{v}(t)$ that evolve according to

$$
c_{v}(t)=\mathrm{e}^{\mathrm{i} \epsilon_{v} t / \hbar} c_{v}(\mathrm{O}) .
$$

While the amplitude $|\Psi|^{2}=\left|\Psi_{\uparrow}\right|^{2}+\left|\Psi_{\downarrow}\right|^{2}$ yields the probability of finding the electron at a given position in space, there is much physical information contained in the full $\Psi$, e.g. the probability of observing a given value of the spin.

In this work we consider only pure states, which are described by a complex-valued wavefunction $\Psi(\mathrm{x})$. In our case $x=(\mathbf{r}, s)$, so $\Psi$ can also be considered a spinor, i.e. a two-component vector $\Psi=\left(\Psi_{\uparrow}, \Psi_{\downarrow}\right)$. Each component of a spinor eigenfunction is proportional to an eigenfunction of the spinless electron with a proportionality coefficient dictated by symmetry. Thus each spinor eigenfunction has two components, each of which is an eigenfunction of the spinless electron with different values of $m$. Given $j$, the upper and lower eigenfunctions have $m=j-1 / 2$ and $m=j+1 / 2$, respectively. One can associate to each spinor a $3 \mathrm{D}$ spin vector $\mathbf{v}$ that points in the local direction of the spin.

Light is emitted when the electron, coupled to an electromagnetic field, performs a transition from a state with quantum numbers $v$ and energy $\in_{v}$ to a state with quantum numbers $v^{\prime}$ and a smaller energy $\in_{v^{\prime}}$ (the reverse transition corresponds to light absorption). The energy of the emitted photon is given by $\in=\epsilon_{v}-\epsilon_{v^{\prime}}$ and is related to its frequency $\omega$ by the Planck relation $\in=\hbar \omega$. Symmetry dictates that the transition can only take place if $v$ and $v^{\prime}$ satisfy the selection rules:

For the spinless electron $v=(n, \ell, m), v^{\prime}=\left(n^{\prime}, \ell^{\prime}, m^{\prime}\right)$, with $\ell-\ell^{\prime}= \pm 1, m-m^{\prime}=0, \pm 1$.

For the electron with spin, $v=(n, \ell, J, j)$, $v^{\prime}=\left(n^{\prime}, \ell^{\prime}, J^{\prime}, j^{\prime}\right)$, with $\ell-\ell^{\prime}= \pm 1, j-j^{\prime}=0, \pm 1$.

Fine-splitting of an emission spectral line corresponds to a superposition of three eigenfunctions: a low-lying eigenfunction with quantum numbers $\left(n_{0}, \ell_{0}, J_{0}\right)$, and two "excited" eigenfunctions with $n_{1,2}=n_{0}, \ell_{1,2}=\ell_{0}+1$ and two different values $J_{1}$ and $J_{2}$ satisfying $J_{1,2}=\ell \pm 1 / 2$.

\section{Acknowledgments}

This material is based upon work supported by the (U.S.) National Science Foundation under Grant Numbers 0821858 , 0855279 and IIS1047678 .

\section{References and Notes}

Unedited references provided by the authors.

1 AlloSphere Research Group, "AlloSystem" (2013): <http://github. com/AlloSphere-Research-Group/AlloSystem>.

2 Xavier Amatriain, JoAnn Kuchera-Morin, Tobias Hollerer and Stephen Travis Pope, "The AlloSphere: Immersive Multimedia for Scientific Discovery and Artistic Exploration," IEEE MultiMedia Vol. 16, No. 2, pp. 64-75, 2009.

3 JoAnn Kuchera-Morin, "Performing in Quantum Space: A Creative Approach to N-Dimensional Computing," Leonardo Vol. 44, No. 5, pp. 462-463, 2011.

4 H.E. White, "Pictorial Representations of the Electron Cloud

for Hydrogen-like Atoms," Phvsical Review Vol. 37, No. 11, pp. 1416$1424,1931$.

5 Bernd Thaller, Visual Quantum Mechanics (Springer TELOS, 2000).

6 Bernd Thaller, Advanced Visual Quantum Mechanics (Springer Science, 2005).

7 Paul Falstad, "Hydrogen Atom Orbital Viewer" (2005): <www. falstad.com/qmatom/>.

8 Dean E. Dauger, "Real-Time Visualizations of Quantum Atomic Orbitals" (2001): <http://daugerresearch.com/orbitals/index.shtml>.

9 Mario Belloni and Wolfgang Christian, "OSP-based Programs for Quantum Mechanics: Time Evolution and ISW Revivals" (2004): $<$ www.phy.davidson.edu/FacHome/mjb/xml_qm/default.html $>$.

10 M. Belloni and W. Christian, "Time development in quantum mechanics using a reduced Hilbert space approach," American Journal of Physics Vol. 76, No. 4, p. 385, 2008. 
11 John Whitney, Digital Harmony: On the Complementarity of Music and Visual Art (Kingsport Press, 1980).

12 Julian Voss-Andreae, "Quantum Sculpture: Art Inspired by the Deeper Nature of Reality," Leonardo Vol. 44, No. 1, pp. 14-20, 2011.

13 H. Schenker, Free Composition (Der Freie Satz): Volume III of New Musical Theories and Fantasies (Schirmer, 1979).

14 Iannis Xenakis, Formalized Music: Thought and Mathematics in Music (Pendragon Press, 1992).

15 Hans Lundmark, "Visualizing Complex Analytic Functions Using Domain Coloring" (2004): <www.mai.liu.se/ alun/complex/ domain_coloring-unicode.html $>$.

16 Siegmund Brandt, Hans Dieter Dahmen and T. Stroh, Interactive Quantum Mechanics: Quantum Experiments on the Computer, Chapter 7 (Springer, 2011).

17 David Joiner, "Visualization of Hydrogen Lesson" (2008): <www. shodor.org/refdesk/Resources/Activities/VisHydrogen/lesson.php $>$.

18 See Thaller [5].

19 See Thaller [6].

20 L.J. Putnam et al., "Immersed in Unfolding Complex Systems," in Beautiful Visualization: Looking at Data through the Eyes of Experts by L.J. Putnam et al. (O’Reilly Media, 2010) 291-309.

\section{Glossary}

eigenfunction-in quantum mechanics, a wavefunction associated with a specific possible value of a physical observable. A general wavefunction can be obtained as a superposition of eigenfunctions.

perceptualization - the mapping of data so that it can be perceived in a meaningful way through human senses, e.g. visualization and sonification.

pure state-a state of a quantum system that contains its fullest specification compatible with the laws of quantum mechanics. It is described by a wavefunction.

Schrödinger equation - the equation describing the evolution of a quantum state. It reads $i \hbar \partial_{\mathrm{t}} \Psi=\hat{H} \Psi$, where $\hat{H}$ is the Hamiltonian operator associated with the energy of the system.

spin-a purely quantum degree of freedom of elementary particles, representing an intrinsic contribution to the total angular momentum. For electrons, the spin assumes the values $\pm \hbar / 2$, where $\hbar$ is the reduced Planck constant.

superposition-an additive mixture of waveforms, typically from the same family of functions. The parts cannot necessarily be recovered fully from the whole. The solutions of the Schrödinger equation are a superposition of eigenfunctions with time-varying coefficients.

wavefunction-the complex-valued function $\Psi(x)$ defined on the configuration space of a quantum system that contains a full description of a pure quantum state. Its evolution is described by the timedependent Schrödinger equation.

Manuscript received 11 March 2013.

ANNOUNCEMENT

\title{
MIT Press Journals Podcasts
}

\author{
The MIT Press Journals Podcast series allows Leonardo authors and contributors to \\ continue the conversation initiated in the pages of the journal in dialogue with each other. \\ Tune in to hear from authors, editors and artists working at the intersection of art, science \\ and technology. The MIT Press Journals Podcast webpage currently hosts a discussion \\ between Jean-Marc Chomaz, Mikael Fernström, Sean Taylor, Annick Bureaud and \\ Roger Malina on their work in the arts and sciences showcased in the article collection, \\ Water Is in the Air: Physics, Politics, and Poetics of Water in the Arts; a conversation \\ inspired by Leonardo e-Book Series release Art and Atoms, featuring Tami Spector, \\ Philip Ball, Kathryn de Ridder-Vignone, Julian Voss-Andreae and Roger Malina \\ on the connections between science-especially chemistry-and art; and more \\ lively, engaging discussions of interest to the art/science community. \\ To learn more: <www. mitpressjournals.org/page/podcasts>.
}




\section{COLOR PLATE D: STUDIES IN COMPOSING HYDROGEN ATOM WAVEFUNCTIONS}

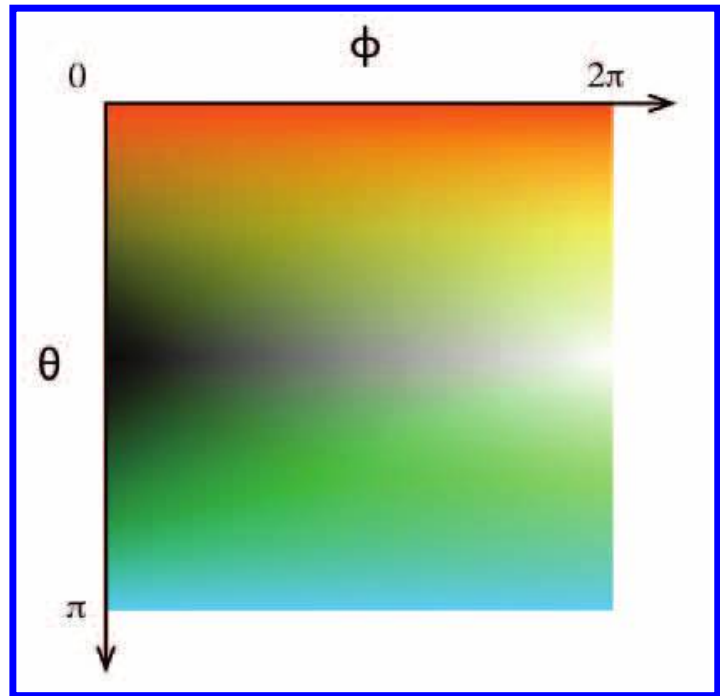

Isosurface color mappings representing the spin vector phases, $\theta$ and $\phi$. Spin up $(\theta=0)$ is orange and spin down $(\theta=\pi)$ is cyan. (C) Lance Putnam) 